\title{
HACIA UNA PERSPECTIVA GLOTOPOLÍTICA Y ETNOGRÁFICA DE LAS LITERACIDADES
}

TOWARDS A GLOTOPOLITIC AND ETHNOGRAPHIC PERSPECTIVE OF LITERACIES

\section{PARA UMA PERSPECTIVA GLOTOPOLÍTICA E ETNOGRÁFICA DAS} LITERACIDADES

Andrey Sebastián Castiblanco Prieto andrey.castiblanco@javeriana.edu.co Instituto Caro y Cuervo

Bogotá- Colombia

Daniel Rudas-Burgos daniel.rudas@caroycuervo.gov.co Instituto Caro y Cuervo

Bogotá - Colombia

Diagramación Sindy Catherine Charcas Ibarra

Fotografia portada Santiago Ardila Acero

Encuentre este artículo en:

http://revistas.uniminuto.edu/index.php/IYD

Para citar este artículo / To cite this article

Castiblanco, A. \& Rudas-Burgos, D. (2021). Hacia una perspectiva glotopolítica

y etnográfica de las literacidades. Inclusión \& Desarrollo, 8 (1), pp 106-116
Fecha de recepción: 18 de septiembre de 2020 Fecha de aceptación: 9 de noviembre de 2020 Fecha de publicación: 1 de enero de 2021

\section{RESUMEN}

Basándose en estudios antropológicos acerca de la lectura y la escritura, este artículo parte de la identificación de un dualismo entre dos perspectivas: la universalista y la relativista. A partir de dos etnografías, una en un club de lectura en un municipio cercano a Bogotá y otra en un programa de alfabetización dirigido a adultos mayores de un barrio periférico de Bogotá, los investigadores identifican en ambos contextos que ambos polos del dualismo se solapan en la práctica. Posteriormente, el artículo problematiza la visión dualista y recomienda una aproximación glotopolítica y etnográfica para abordar la enseñanza de lectura y escritura.

Palabras clave: glotopolítica, sociolingüística, política lingüística, antropología de la educación, enseñanza de la lectura, enseñanza de la escritura; alfabetización de adultos, promoción de lectura. 


\section{ABSTRACT}

Based on anthropological studies about reading and writing, this article starts from the identification of a dualism between two perspectives: universalist and relativist. From two ethnographies, one in a reading club in a municipality near Bogotá and another in a literacy program for older adults in a suburban neighborhood of Bogotá, researchers identify in both contexts that both poles of dualism overlap in practice. Subsequently, the paper problematizes the dualistic view and recommends a glotopolitic and ethnographic approach to teaching reading and writing.

Keywords: glotopolitics, sociolinguistics, linguistic policy, anthropology of education, teaching of reading, teaching of writing, adult literacy, promotion of reading.

\section{SUMÀRIO}

Baseando-se em estudos antropológicos acerca da leitura e da escrita, este artigo parte da identificação de um dualismo entre duas perspectivas: a universalista e a relativista. A partir de duas etnografias, uma em um clube de leitura em um município próximo a Bogotá e outra em um programa de alfabetização dirigido a adultos maiores de um bairro periférico de Bogotá, os pesquisadores identificam em ambos os contextos que ambos os pólos do dualismo se sobrepõem na prática. Posteriormente, o artigo problematiza a visão dualista e recomenda uma aproximação Glotopolítica e etnográfica para abordar o ensino de leitura e escrita.

Palavras chave: Política, sociolinguística, política linguística, antropologia da educação, ensino da leitura, ensino da escrita, alfabetização de adultos, promoção da leitura. 
Este artículo, que está planteado desde una perspectiva glotopolítica, parte de dos ideas estrechamente relacionadas. La primera es que todo acto lingüístico es político (Joseph, 2006/2017; Del Valle, 2017) y, por ende, la comprensión de la enseñanza o la promoción de cualquier práctica lingüística debe prestar especial atención a su aspecto político. La segunda es que todo discurso metalingüístico es igualmente político y, por ende, tiene unos efectos tanto en la práctica lingüística de las personas comoen las políticas gubernamentales acerca del lenguaje (Silverstein, 1993; Rosa \& Burdick, 2016).

Basándose en estas dos ideas, este artículo comienza por presentar dos discursos antropológicos opuestos acerca de la lectura y la escritura, que denominaremos como la perspectiva relativista y la perspectiva universalista. Posteriormente, presentamos los resultados de dos etnografías realizadas bajo la perspectiva relativista: una en un club de lectura dirigido a jóvenes y otra en un programa de alfabetización dirigido a adultos mayores. Apartir de estas etnografías, el artículo plantea que no es posible comprender los casos observados solamente desde la perspectiva relativista, pues en la práctica ambas perspectivas se solapan. Finalmente, el artículo invita a utilizar una perspectiva etnográfica y glotopolítica para comprender, promover y enseñar prácticas de lectura y escritura.

\section{Antecedentes}

Es posible establecer un dualismo en las investigaciones antropológicas sobre la lectura y la escritura entre una perspectiva universalista y una relativista. Aun cuando las conclusiones de ambas perspectivas surgen del estudio de casos concretos, la perspectiva universalista defiende que la lectura siempre, sin importar el contexto, produce unos efectos culturales y cognitivos, mientras que la perspectiva relativista defiende que los efectos de la lectura no son universalizables sino contextuales (Bartlett et al., 2011; Rudas-Burgos \& Castiblanco, 2018).

La perspectiva universalista estudia principalmente la escritura alfabética occidental y centra la mirada en los efectos que esta ha traído al desarrollo de las sociedades donde se usa. Para esta perspectiva, lo importante no es comprender la práctica de la lectura y la escritura en cuanto tal, sino identificar y argumentar a favor de unos beneficios, usualmente asociados a valores occidentales, que el alfabeto trae a las personas y sociedades letradas. Así, en lo que refiere a las personas letradas, los beneficios que desde esta perspectiva suelen relacionarse con la lectura y la escritura son que estas personas desarrollan pensamiento crítico, abstracto y estético; fortalecen sus valores democráticos y aumentan sus posibilidades económicas y laborales. En lo que refiere a las sociedades letradas, los beneficios personales son transferidos para argumentar que la alfabetización y la promoción de lectura fortalecen la democracia y traen un desarrollo social y económico a la mayoría de naciones del mundo (Goody, 2000; Graff \& Duffy, 2008; United Nations Educational, Scientific and Cultural Organization, 2006).

Los trabajos hechos desde la perspectiva universalista son considerados dentro de algunos sectores de la antropología como etnocéntricos, colonialistas y deterministas (Jouve-Martín, 2009). A estos se les critica que establecen una jerarquía entre escritura y oralidad que sitúa al "no lector" como alguien rezagado en el tiempo. También se critica que el elogio a los beneficios universales de la lectura y la escritura tiene un efecto colateral: el rechazo y la estigmatización arbitraria de las sociedades y las personas que desde una óptica occidental son calificadas como "no lectoras" (Farr, 2009; Papen, 2005; Street, 2009). 
Es por ello que la perspectiva relativista, que es mucho más sensible a la diversidad cultural, defiende que no hay beneficios universales de la lectoescritura y que sus efectos deben identificarse en su uso, en cada contexto particular (Street, 2001). Así, esta perspectiva no solo estudia la escritura alfabética occidental sino que también reconoce lo que ha llamado escrituras vernaculares, como graffitis, tejidos, peinados, tatuajes, entre otras (véase por ejemplo Garcia etal., 2008; Kafle \& Canagarajah, 2015; Mahiri, 2008; Rosario-Ramos \& Johnson, 2014). Desde esta perspectiva, los discursos, las ideologías y las relaciones de poder tienen un papel preponderante.

\section{Metodología}

Los estudios antropológicos que se enmarcan en la perspectiva relativista, que fue la que adoptamos para realizar la investigación, ha ideado conceptos que permiten describir y caracterizar las formas en las que leer y escribir cobran sentido en contextos particulares. Dos de esos conceptos son los eventos y las prácticas de lectura y escritura.

Los eventos son una categoría descriptiva que da cuenta de ocasiones en las que el lenguaje escrito es esencial para la interacción de los participantes. Este concepto aporta una unidad de análisis y permite caracterizar los diferentes usos y valoraciones que la gente le da a la lectura y a la escritura. Por su parte, las prácticas de lectura y escritura sirven para ubicar los eventos documentados en los marcos que les dan sentido, es decir, los eventos se relacionan con las instituciones, las relaciones de poder $y$, en general, con los contextos sociohistóricos particulares que los posibilitan (Niño-Murcia, 2009).

Con el fin de documentar los eventos y construir las prácticas, nosotros usamos el método etnográfico, el cual entendemos como un análisis retrospectivo a posteriori que permite convertir la experiencia, el trabajo de campo, las notas y la memoria en datos (Ingold, 2014). En este sentido, realizamos observación participante intermitentemente desde el 2015 hasta el 2018 en cada uno de los casos estudiados. La observación participante es una técnica en la que el investigador debe prestar atención y relacionarse con las personas con el fin de dejar cualquier punto de vista previamente adoptado (pp. 388-389); y registrar sistemáticamente, usualmente a partir de la toma de notas, la experiencia del trabajo de campo (Guber, 2001, p. 57). Con la información recolectada, realizamos entrevistas semiestructuradas, las cuales se usaron con el fin de focalizar y profundizar en la experiencia obtenida durante la observación participante (p. 92). Para terminar, conversamos de las conclusiones del estudio con los miembros de ambos programas, esto con el fin de incluir sus impresiones acerca de la investigación en la investigación.

\section{Resultados}

Después de realizar nuestro trabajo de campo, presentamos dos aportes. El primero es que, como es de esperarse, en la práctica los dos extremos del dualismo se solapan, es decir, el conjunto de ideas y prácticas de los participantes sobre la lectura y la escritura no puede asociarse enteramente ni a lo universalista ni a lo relativista, sino que se corresponde algunas veces a una perspectiva y otras a la otra. El segundo es que hoy la mirada se aleja del polo universalista y corre el riesgo de caer de lleno en el relativista. Discutiremos este segundo hallazgo en la sección de conclusiones.

Por un lado, el pensum del programa de alfabetización fue creado intuitivamente por voluntarios de la organización Colombia Crece. Este pensum enseñaba y evaluaba, entre otros temas, gramática (puntuación) y ortografía 
y también promovía la producción de textos periodísticos y literarios (lectura de noticias de Colombia y escritura de cuentos y poemas). Los estudiantes del programa eran en su mayoría adultos mayores provenientes de diversas zonas rurales del país. Ellos, que ya tenían una gran dificultad en el dominio de la técnica de la escritura y de la lectura, tenían otro gran obstáculo en aprender las normas de ortografía y puntuación, que son arbitrarias no solamente por la naturaleza no motivada de las mismas en términos lingüísticos (Saussure, 1916/1945), sino porque históricamente las instituciones normativas de la lengua española se han resistido a simplificaciones que facilitarían su aprendizaje (Villa, 2015). Además, a los estudiantes del programa no les resultaba familiar la escritura de cuentos ni les era sencillo hacer una lectura crítica de periódicos mientras ponían todo su esfuerzo en descifrar el código escrito. Ante estas dificultades, el pénsum del programa se adaptó y dejó de evaluar la ortografía y la gramática y promovió que los ejercicios de lectura y escritura se basaran en géneros más próximos a los estudiantes como relatos de espantos, textos religiosos, recetas de cocina o memorias de la infancia o de la vida cotidiana.

Por otro lado, en Guatavita, el club de lectura fue creado por un par de profesores que querían promover la lectura de una manera alternativa a como se hacía en el colegio público donde enseñaban. En el club de lectura se leía literatura de todo tipo, pero también se veían videos, fotografías, se grafiteaba, se dibujaba, se cocinaba, se recorría el territorio y se discutían los temas de interés de quienes asistían al Club; desde la memoria de Guatavita pasando por fantasmas hasta teorías de la conspiración. Cuando preguntamos por los objetivos del Club a los profesores, uno asoció la lectura con la creación de una ciudadanía volcada al amor por lo intelectual y lo estético, mientras que la otra profesora manifestó no tener un interés pedagógico explícito ni particular con este.
Basados en lo anterior, nosotros concluimos que ambas perspectivas se solapan porque, por una parte, en el caso del programa de alfabetización, conviven prácticas en las que se reconoce el contexto de los participantes con sistemas de validación basados en estándares universales $\mathrm{y}$, por otra parte, en el caso del club de lectura, conviven la inclusión de elementos del contexto y la exploración de formas alternativas de lectura y escritura con la idea de que estas favorecen la creación de un pensamiento intelectual -que es asociado generalmente al pensamiento crítico- y el desarrollo de una cierta sensibilidad por lo estético.

\section{Discusión}

¿Por qué decimos que se corre el riesgo de caer en el relativismo? Para Pierre Bourdieu, la dificultad de las ciencias sociales radica en que siempre se navega entre dos escollos, entre dualismos, y cuando uno esquiva el de la izquierda, puede chocar con el de la derecha. En ese sentido, Bourdieu (1997, pp. 46-47) considera que, dependiendo del curso del tiempo, a veces es necesario criticar el polo al que más se tiende con el fin de evitar caer de nuevo en un polo del dualismo.

Como hemos visto, la teoría social acerca de la lectura y la escritura no es la excepción: su mirada se mueve entre el universalismo $y$ el relativismo. Los primeros estudios antropológicos acerca de la lectura y la escritura tendían hacia lo universalista (Goody \& Watt, 1963; Ong, 1982/1987). Luego, desde la antropología, hubo una serie de trabajos que criticaron esta perspectiva y abogaron por el reconocimiento de otros tipos de lectura, como los los tejidos, los peinados, los graffitis, entre otros (Kafle \& Canagarajah, 2015; Street, 1984; Kiramba, 2017). Así, estos estudios hicieron que la mirada se alejara de lo universalista y se acercara a lo relativista. Por ello, siguiendo a 
Bourdieu, señalaremos algunos puntos ciegos de la perspectiva relativista identificados en los casos analizados para luego hacer una observación general a las políticas realizadas desde esta perspectiva.

Acerca de nuestros casos analizados, vimos, por un lado, que en el programa de alfabetización se renunció a la corrección de ortografía y la gramática. Si bien fue una decisión razonable, pues la mayoría de sus estudiantes son adultos mayores interesados en una lectura funcional (tomar el bus, pagar un servicio público, buscar direcciones, entre otras) esta decisión podría influir negativamente en la minoría de adultos y jóvenes que asisten al programa de alfabetización y que pueden jugarse un trabajo, una calificación o su prestigio en la ortografía. $\mathrm{Si}$ bien puede hacerse que desaparezca la mirada evaluadora y estigmatizadora de los estándares universales en un programa puntual de alfabetización, aún falta mucho trabajo para hacer que la discriminación basada en el uso del lenguaje sea tan notoria y sancionada como lo son la discriminación racial o la discriminación de género (Cameron, 1995/2005; Lippi-Green, 1994; Rodríguez, 2018).

En el mismo sentido, en el club de lectura, leer no perseguía un objetivo particular, incluso el club de lectura a veces giraba alrededor de otras actividades que no se conectaban necesariamente con la lectura y la escritura. Esto hace que, aunque en el club se reconocieran diferentes tipos de lectura, se difuminara la razón para hacerlo y hasta el acto mismo de leer en sus actividades. Esto cobra importancia si entendemos que todo acto de lenguaje es político y que, por ende, todo acto de promoción de lectura lo es (Joseph, 2006/2017). En todas estas situaciones, nuestras ideologías acerca del lenguaje y nuestros intereses, a veces conscientes a veces inconscientes, nos llevan a pugnar por la promoción de ciertas ideas, a motivar a otros, a transformarlos de maneras que no esperamos. En este sentido, dado que no es posible ejercer actos no políticos, conviene tener claro cuáles son los propósitos de nuestras acciones más pretendidamente libres.

Este acercamiento al polo relativista no solo sucede en los dos casos que mencionamos, sino que es una tendencia en las políticas acerca de lectura, por lo menos, en las naciones occidentales. Wickens \& Sandlin (2007), al analizar las políticas de alfabetización de la Unesco y del Banco Mundial, encuentran que estas han pasado de un discurso neocolonial -que asocia la alfabetización a la economía y la productividad individual - a un discurso interesado en la gobernanza local -que asocia la alfabetización a las relaciones sociales y a la cultura-. A pesar de ello, estas autoras concluyen que, por los objetivos de los programas de alfabetización y las restricciones de los fondos que brindan, estas políticas no pueden desligarse del proyecto neocolonial.

Es por ello que nosotros consideramos que debemos ser especialmente rigurosos con las políticas de promoción de lectura planteadas desde una perspectiva relativista. No debemos perder de vista que el multiculturalismo despolitizado es la posición ideológica dominante del capitalismo global (Zizek, 2008). Esto hace que el discurso del multiculturalismo tenga la capacidad de hacer pasar por neutras, es decir, fácilmente aceptables e incuestionables, políticas y programas que merecen revisiones más detalladas. También es importante recordar que históricamente el colonialismo se ha valido de las lenguas de los dominados para implementar su proyecto colonial (Poblete, 2016; Rama, 1984/1998; Mignolo \& Walsh, 2018), por lo que fomentar y reconocer diversas formas de lectura y escritura no es una razón suficiente para justificar cualquier política de promoción de lectura o escritura. 


\section{Conclusiones}

Debido a que el uso del lenguaje es inherentemente político (Joseph, 2006/2017), todo acto de promoción de lectura tiene unas implicaciones políticas. En este sentido, nuestra crítica tiene el interés de suscitar una mayor reflexión sobre cualquier acto de promoción de lectura, más aún sobre aquellos que se basan en los supuestos de que la lectura nos hace por sí misma "mejores personas". Esto con el ánimo de tener cada vez más claro cómo definir los horizontes de la promoción de la lectura y escritura en Colombia, que, sin lugar a dudas, deben ser horizontes marcados por el disenso, el consenso y por una conciencia glotopolítica, esto es, por la conciencia de que los discursos ( $y$, más particularmente, los juicios y estereotipos que se asocian al uso del lenguaje) tienen implicaciones en el acceso a recursos, la toma de decisiones y la participación política (Del Valle, 2015, 2017).

Para José del Valle: "La estrategia clave es abogar por una educación lingüística basada en los principios de una teoría glotopolítica del lenguaje" (Del Valle, 2020). Para nosotros, esto significa que los diseños pedagógicos para la enseñanza de la lectura y la escritura, ya sea en contextos escolares como no escolares, se beneficiarían de tener en cuenta que las perspectivas universalistas y las relativistas sobre la lectura y la escritura están presentes en las ideologías tanto de los estudiantes como de los docentes y otros agentes institucionales. Como estas perspectivas entran en tensión en la práctica de forma particular en cada caso, es necesario indagar por estas a través de un trabajo etnográfico, que puede ser conducido por docentes y actores locales (Rogers, 2005; Street et al., 2006), y para el que sugerimos usar referentes glotopolíticos. De igual manera, dado que no es posible prever todas las implicaciones políticas de una cierta práctica lingüística, es importante promover una conciencia metalingüística crítica en estudiantes y docentes (García y Kleifgen, 2019), así como en la sociedad en general.

\section{Declaraciones}

Reconocimientos: Por permitirnos realizar el trabajo etnográfico, agradecemos a los miembros del Cub de Lectura Ulular y a los miembros del programa de Alfabetización de ColombiaCrece. También agradecemos por las retroalimentaciones a los miembros del semillero prácticas locales de lectura y escritura, de la línea de Glotopolítica del Instituto Caro y Cuervo, y a los miembros del semillero de estudios estructuralistas de la Pontificia Universidad Javeriana. Por último, agradecemos a la Corporación Universitaria Minuto de Dios por fomentar y reconocer la investigación de los semilleros del país.

Financiamiento: este proyecto fue financiado por el Instituto Caro y Cuervo en el marco del proyecto "Prácticas de escritura y lectura en Cundinamarca".

Disponibilidad de datos y materiales: para contactar a los autores puede remitirse a los correos andrey.castiblanco@javeriana.edu.co y daniel.rudas@caroycuervo.gov.co

Contribución de autores: la investigación fue realizada de manera conjunta por Daniel Rudas-Burgos y Andrey Castiblanco en el marco de un proceso formativo en el Semillero lectura y escritura como prácticas culturales del Instituto Caro y Cuervo, cuyo tutor es Daniel Rudas-Burgos.

Aprobación ética y consentimiento de los participantes: el Instituto Caro y Cuervo aprobó el proyecto general "Prácticas de escritura y lectura en Cundinamarca" y sus consideraciones éticas en 2015. Toda la investigación se hizo 
siguiendo las normas consuetudinarias de los contextos donde se desarrolló la investigación, todas las personas entrevistadas firmaron un consentimiento informado en el que se les informó del propósito de la investigación, sus beneficios y riesgos. Además, los resultados de la investigación se conversaron con los participantes con el fin de incluir sus percepciones de los resultados de esta.

Conflictos de intereses: no consideramos que existan conflictos de intereses. 


\section{Referencias bibliográficas}

Bartlett, L., López, D., Vasudevan, L., \& Warriner, D. (2011). The Anthropology of Literacy. En B. Levinson \& M. Pollock (Eds.), A Companion to the Anthropology of Education (pp. 154-176). Wiley-Blackwell.

Bourdieu, P. (1997). Conversación: El oficio del sociólogo. En I. Jiménez (Ed.), Capital cultural, escuela y espacio social (pp. 39-57). Siglo XXI.

Cameron, D. (2005). Verbal Hygiene: The Politics of Language. Routledge. (Publicado originalmentne en 1995)

Del Valle, J. (2015). Lenguaje, política e historia: Ensayo introductorio. En J. Del Valle (Ed.), Historia política del español: La creación de una lengua (pp. 3-23). Aluvión.

Del Valle, J. (2017). La perspectiva glotopolítica y la normatividad. Anuario de Glotopolítica, 1, 17-39. https://glotopolitica.files.wordpress.com/2018/04/aglo20selection.pdf\#page=15 Del Valle, J. (2020). Entrevista: Glotofobia [En prensa].

Farr, M. (2009). Ideologías de la alfabetización: Prácticas locales y definiciones culturales. En J. Kalman \& B. Street (Eds.), Lectura, escritura y matemáticas como prácticas sociales: Diálogos con América Latina (pp. 99-111). Siglo XXI: CREFAL.

Garcia, O., Bartlett, L., \& Kleifgen, J. (2008). From biliteracy to pluriliteracies. En P. Auer \& L. Wei (Eds.), Handbook of Multilingualism and Multilingual Communication (pp. 207-228). De Gruyter.

García, O., \& Kleifgen, J. (2019). Translanguaging and Literacies. Reading Research Quarterly, 0(0), 1-19. https://doi.org/doi:10.1002/rrq.286

Gee, J. P. (1989). Literacy, Discourse, and Linguistics: Introduction. The Journal of Education, 171(1), 5-176.

Goody, J. (2000). The Power of the Written Tradition. Smithsonian Institution Press.

Goody, J., \& Watt, I. (1963). The Consequences of Literacy. Comparative Studies in Society and History, 5(3), 304-345. https://doi.org/10.1017/S0010417500001730

Graff, H., \& Duffy, J. (2008). Literacy Myths. En B. Street \& N. Hornberger (Eds.), Literacy (Encyclopedia of Language and Education, Vol. 2, pp. 41-52). Springer.

Guber, R. (2001). La etnografía: Método campo y reflexividad. Grupo Editorial Norma.

Heath, S. B. (2009). Ways With Words. Language Life, and Work in Communities and Classrooms (Kindle 4 Version). Cambridge University Press. Amazon.com (Publicado originalmente en 1983)

Ingold, T. (2014). That's enough about ethnography! Journal of ethnographic theory, 4(1), 383-395. https://doi.org/10.14318/hau4.1.021

Joseph, J. E. (2017). De cómo la política impregna el lenguaje (y viceversa): Visión panorámica (J. Del Valle \& L. Villa, Trads.). Anuario de Glotopolítica, 1, 279-312. https://glotopolitica.files.wordpress.com/2018/04/aglo20selection.pdf\#page $=277$ (Publicado originalmente en 2006)

Jouve-Martín, J. (2009). Epílogo. Escritura, hegemonía y subalternidad: De los New literacy studies (NLS) a los Latin american literacy studies (LALS), and back. En J. Kalman \& B. 
Street (Eds.), Lectura, escritura y matemáticas como prácticas sociales: Diálogos con América Latina (pp. 387-398). Siglo XXI: CREFAL.

Kafle, M., \& Canagarajah, S. (2015). Multiliteracies, Pedagogies, and Academic Literacy. En W. Wright, S. Boun, \& O. García (Eds.), Handbook of Bilingual Education (pp. 241-252). Wiley-Blackwell.

Kiramba, L. K. (2017). Multilingual Literacies: Invisible Representation of Literacy in a Rural Classroom. Journal of Adolescent \& Adult Literacy. https://doi.org/10.1002/jaal.690 Lippi-Green, R. (1994). Accent, Standard Language Ideology, and Discriminatory Pretext in the Courts. Language in Society, 23(2), 163-198. http://www.jstor.org/stable/4168513 Mahiri, J. (2008). Literacies in the Lives of Urban Youth. En B. Street \& N. Hornberger (Eds.), Literacy (Encyclopedia of Language and Education, Vol. 2, pp. 299-307). Springer. Mignolo, W. (1995). The darker side of the renassaince. The University of Michigan Press. Mignolo, W. D., \& Walsh, C. E. (2018). On Decoloniality: Concepts, Analytics, Praxis. Duke University Press.

Niño-Murcia, M. (2009). Prácticas letradas exuberantes en la periferia de la república de las letras. En J. Kalman \& B. Street (Eds.), Lectura, escritura y matemáticas como prácticas sociales: Diálogos con América Latina (pp. 115-129). Siglo XXI: CREFAL.

Ong, W. J. (1987). Oralidad y escritura: Tecnologías de la palabra (Á. Scherp, Trad.). Fondo de Cultura Económica. (Original work published 1982)

Papen, U. (2005). Literacy and development: What works for whom? Or, how relevant is the social practices view of literacy for literacy education in developing countries? International Journal of Educational Development, 25, 5-17. https://doi.org/10.1016/j.ijedudev.2004.05.001 Poblete, J. (2016). De la lectura como práctica histórica en América Latina: La primer época colonial y el siglo XIX. Cuadernos de literatura 20, 39, 57-94. https://doi.org/10.11144/Javeriana.cl20-39.Ipha

Rama, Á. (1998). La ciudad letrada. Arca. (Publicado originalmente en 1984)

Rodríguez, A. C. (2018). Actitudes e ideologías lingüísticas de docentes de español: Entre la corrección y el valor de la diversidad. Análisis, 50(92), 95-117. https://revistas.usantotomas. edu.co/index.php/analisis/article/view/3701

Rogers, A. (2005). Training adult literacy educators in developing countries. Paper commissioned for the EFA Global Monitoring Report 2006, Literacy for Life. http://portal.unesco.org/ education/fr/files/43487/11315414901Rogers_A.doc/Rogers_A.doc

Rosa, J., \& Burdick, C. (2016). Language Ideologies. En O. Garcia, N. Flores, \& M. Spotti (Eds.), The Oxford Handbook of Language and Society. Oxford University Press. http://dx. doi.org/10.1093/oxfordhb/9780190212896.013.15

Rosario-Ramos, E., \& Johnson, L. R. (2014). Communities as Counter-Storytelling (Con) Texts. The Role of Community-Based Educational Institutions in the Development of Critical Literacy and Transformative Action. En J. Zacher-Pandya \& J. Ávila (Eds.), Moving Critical Literacies Forward. A New Look at Praxis Across Contexts. Routledge.

Rudas-Burgos, D., \& Castiblanco, A. (2018). Perspectivas universalistas y relativistas de la 
lectura y la escritura en dos casos en Colombia: Una mirada antropológica. Análisis, 50(92), 15-34. https://revistas.usantotomas.edu.co/index.php/analisis/article/view/3657

Saussure, F. de. (1945). Curso de lingüística general (C. Bally \& A. Séchehaye, Eds.; A. Alonso, Trad.). Losada. ( Publicado originalmente en 1916)

Silverstein, M. (1993). Metapragmatic Discourse and Metapragmatic Function. En J. A. Lucy (Ed.), Reflexive Language: Reported Speech and Metapragmatics (pp. 33-58). Cambridge University Press.

Street, B. (1984). Literacy in theory and practice. Cambridge University Press.

Street, B. (2001). Introduction. En B. Street (Ed.), Literacy and development: Ethnographic perspectives (pp. 1-19). Routledge.

Street, B. (2009). Perspectivas etnográficas y políticas sobre cultura escrita: El poder de nombrar y definir. En J. Kalman \& B. Street (Eds.), Lectura, escritura y matemáticas como prácticas sociales: Diálogos con América Latina (pp. 84-98). Siglo XXI: CREFAL.

Street, B., Rogers, A., \& Baker, D. (2006). Adult teachers as researchers: Ethnographic approaches to numeracy and literacy as social practices in South Asia. Convergence, 39(1), 31-44.

United Nations Educational, Scientific and Cultural Organization. (2006). Education for All Global Monitoring Report: Literacy for Life. United Nations Educational, Scientific and Cultural Organization (UNESCO). http://unesdoc.unesco.org/images/0014/001416/141639e.pdf Villa, L. (2015). La oficialización del español en el siglo XIX: la autoridad de la Academia. En J. Del Valle (Ed.), Historia política del español: La creación de una lengua (pp. 107-121). Aluvión.

Wickens, C., \& Sandlin, J. (2007). Literacy for what? Literacy for whom? The politics of literacy education and neocolonialism in UNESCO -and World bank- sponsored literacy programs. Adult education quarterly, 57(4), 275-292. https://doi.org/10.1177/0741713607302364 Zizek, S. (2008). En defensa de la intolerancia. Sequitur. 